\title{
Application of SRAP fingerprinting for analysis of Lupinus angustifolius cDNA
}

\author{
E. Sysoliatin*, N. Anisimova, A. Kilchevsky \\ Institute of Genetics and Cytology, NAS of Belarus, Minsk, Belarus \\ *e-mail: E.Sysoliatin@igc.by
}

Key words: cDNA fingerprinting, differential expression, disease response

Motivation and Aim: Narrow-leaved lupine (Lupinus angustifolius L.) is a valuable legume crop capable of nitrogen fixation. There is a need to expand a list of molecular markers of the genes of economically valuable traits and to study the molecular mechanisms that determine the traits. SRAP (sequence-related amplified polymorphism) is based on the amplification of open reading frames. This method proved to be suitable in differential expression studies of various plant cultures [1]. The aim of this work was to assess the feasibility of using SRAP for cDNA analysis of narrow-leaved lupine samples.

Methods and Algorithms: RNA was isolated from the roots of ten-day seedlings of narrow-leaved lupine varieties Aschadny, Yorrel, Frost, Nemchinovsky 846. There were two groups of seedlings: intact and exposed to Fusarium culture. cDNA was synthesized on the RNA matrix and was used in a series of PCR reactions with 12 combinations of 3 forward (Me8, f12, f16) and 4 reverse (Em5, Em12, r14, r9) SRAP primers [2, 3]. SRAP fragments associated with resistant seedlings were isolated and sequenced. Their putative function was assigned based on alignment to known sequences and conserved domain search.

Results: The results showed that 12 primer combinations produced a total of 234 clear bands. The proportion of polymorphic bands varied in the range from 66,7 to $100 \%$. The SRAP fragment (f12-r14-290) associated with seedlings resistant to Fusarium isolates (Frost, Aschadny) were found. Fragment sequencing and subsequent analysis showed the presence of SNARE domain, which is characteristic of syntaxin-binding protein (STBP).

Conclusion: The results obtained indicate that the SRAP method is suitable for the analysis of narrow-leaved lupine cDNA and can be used to study the differential gene expression of this culture. The SRAP fragment associated with resistant seedlings showed the presence of SNARE domain, characteristic of STBP involved in exocytosis and mechanisms of plant nonspecific resistance to pathogens.

Acknowledgements: Supported by the SPSR "Biotechnology", subprogram "Structural and functional genomics", task: "Biotechnology 2.02. Evaluation of narrow-leaved and yellow lupine gene pools by resistance to fungal diseases based on DNA-marking".

\section{References}

1. Que Y. et al. (2012) cDNA-SRAP and Its Application in Differential Gene Expression Analysis: A Case Study in Erianthus arundinaceum. Journal Biomedicine Biotechnology. Article ID 390107.

2. Mutlu N. et al. (2008) Development of SRAP, SRAP-RGA, RAPD and SCAR markers linked with a Fusarium wilt resistance gene in eggplant. Theor Appl Genet. 117(8):1303-1312.

3. Ma J.-X. et al. (2013) Genetic diversity of wild Medicago sativa by sequence-related amplified polymorphism markers in Xingjiang region, China. Pak. J. Bot. 45(6):2043-2050. 